\title{
HERMITE-HADAMARD TYPE INEQUALITIES FOR FUNCTIONS WHEN A POWER OF THE ABSOLUTE VALUE OF THE FIRST DERIVATIVE IS P-CONVEX
}

\author{
A. BARANI ${ }^{\otimes}$ and S. BARANI
}

(Received 31 August 2011)

\begin{abstract}
In this paper we extend some estimates of the right-hand side of a Hermite-Hadamard type inequality for functions whose derivatives' absolute values are $P$-convex. Applications to the trapezoidal formula and special means are introduced.
\end{abstract}

2010 Mathematics subject classification: primary 26A51; secondary 26D15.

Keywords and phrases: Hermite-Hadamard inequality, $P$-convex, trapezoidal formula, special means.

\section{Introduction}

Let $I=[c, d]$ be an interval on the real line $\mathbb{R}$, let $f: I \rightarrow \mathbb{R}$ be a convex function and let $a, b \in[c, d], a<b$. We consider the well-known Hermite-Hadamard inequality

$$
f\left(\frac{a+b}{2}\right) \leq \frac{1}{b-a} \int_{a}^{b} f(x) d x \leq \frac{f(a)+f(b)}{2} .
$$

Both inequalities hold in the reverse direction if $f$ is concave (see [12]). The classical Hermite-Hadamard inequality provides estimates of the mean value of a continuous convex function $f:[a, b] \rightarrow \mathbb{R}$. We note that the Hermite-Hadamard inequality may be regarded as a refinement of the concept of convexity, as follows easily from Jensen's inequality. The Hermite-Hadamard inequality for convex functions has received renewed attention in recent years and a remarkable variety of refinements and generalisations has been found; see, for example, [4-7] and references therein.

Dragomir and Agarwal in [9] used the following lemma to prove Theorems 1.2 and 1.3 .

LEMMa 1.1. The following equation holds true:

$$
\frac{f(a)+f(b)}{2}-\frac{1}{b-a} \int_{a}^{b} f(x) d x=\frac{b-a}{2} \int_{0}^{1}(1-2 t) f^{\prime}(t a+(1-t) b) d t .
$$

(C) 2011 Australian Mathematical Publishing Association Inc. 0004-9727/2011 \$16.00 
TheOREM 1.2. Assume that $a, b \in \mathbb{R}$ with $a<b$ and $f:[a, b] \rightarrow \mathbb{R}$ is a differentiable function on $(a, b)$. If $\left|f^{\prime}\right|$ is convex on $[a, b]$ then the following inequality holds true:

$$
\left|\frac{f(a)+f(b)}{2}-\frac{1}{b-a} \int_{a}^{b} f(x) d x\right| \leq \frac{(b-a)\left(\left|f^{\prime}(a)\right|+\left|f^{\prime}(b)\right|\right)}{8} .
$$

Theorem 1.3. Assume that $a, b \in \mathbb{R}$ with $a<b$ and $f:[a, b] \rightarrow \mathbb{R}$ is a differentiable function on $(a, b)$. Assume that $p \in \mathbb{R}$ with $p>1$. If $\left|f^{\prime}\right|^{p /(p-1)}$ is convex on $[a, b]$ then the following inequality holds true:

$$
\begin{aligned}
& \left|\frac{f(a)+f(b)}{2}-\frac{1}{b-a} \int_{a}^{b} f(x) d x\right| \\
& \quad \leq \frac{b-a}{2(p+1)^{1 / p}} \cdot\left(\frac{\left|f^{\prime}(a)\right|^{p /(p-1)}+\left|f^{\prime}(b)\right|^{p /(p-1)}}{2}\right)^{(p-1) / p} .
\end{aligned}
$$

In [12] Pečarić et al. proved the following theorem.

Theorem 1.4. Let $f: I \rightarrow \mathbb{R}$ be a differentiable function on $I^{\circ}, a, b \in I^{\circ}$ with $a<b$. If $\left|f^{\prime}\right|^{q}$ is convex on $[a, b]$ for $q \geq 1$, then the following inequality holds:

$$
\left|\frac{f(a)+f(b)}{2}-\frac{1}{b-a} \int_{a}^{b} f(x) d x\right| \leq \frac{(b-a)}{4}\left(\frac{\left|f^{\prime}(a)\right|^{q}+\left|f^{\prime}(b)\right|^{q}}{2}\right)^{1 / q} .
$$

Recall that the function $f:[a, b] \rightarrow \mathbb{R}$ is said to be quasiconvex if, for every $x, y \in[a, b]$,

$$
f(t x+(1-t) y) \leq \max \{f(x), f(y)\} \quad \text { for all } t \in[0,1] .
$$

Ion in [10] presented some estimates of the right-hand side of a Hermite-Hadamard type inequality in which some quasiconvex functions are involved. The main results of [10] are given by the following theorems.

Theorem 1.5. Assume that $a, b \in \mathbb{R}$ with $a<b$ and $f:[a, b] \rightarrow \mathbb{R}$ is a differentiable function on $(a, b)$. If $\left|f^{\prime}\right|$ is quasiconvex on $[a, b]$ then the following inequality holds true:

$$
\left|\frac{f(a)+f(b)}{2}-\frac{1}{b-a} \int_{a}^{b} f(x) d x\right| \leq \frac{(b-a) \max \left\{\left|f^{\prime}(a)\right|,\left|f^{\prime}(b)\right|\right\}}{4} .
$$

Theorem 1.6. Assume that $a, b \in \mathbb{R}$ with $a<b$ and $f:[a, b] \rightarrow \mathbb{R}$ is a differentiable function on $(a, b)$. Assume that $p \in \mathbb{R}$ with $p>1$. If $\left|f^{\prime}\right|^{p /(p-1)}$ is quasiconvex on $[a, b]$ then the following inequality holds true:

$$
\begin{aligned}
& \left|\frac{f(a)+f(b)}{2}-\frac{1}{b-a} \int_{a}^{b} f(x) d x\right| \\
& \quad \leq \frac{b-a}{2(p+1)^{1 / p}}\left(\max \left\{\left|f^{\prime}(a)\right|^{p /(p-1)},\left|f^{\prime}(b)\right|^{p /(p-1)}\right\}\right)^{(p-1) / p} .
\end{aligned}
$$


On the other hand, Dragomir et al. in [7] defined the following class of functions.

Definition 1.7. Let $I \subseteq \mathbb{R}$ be an interval. The function $f: I \rightarrow \mathbb{R}$ is said to belong to the class $P(I)$ (or to be $P$-convex) if it is nonnegative and, for all $x, y \in I$ and $\lambda \in[0,1]$, satisfies the inequality

$$
f(\lambda x+(1-\lambda) y) \leq f(x)+f(y) .
$$

Note that $P(I)$ contain all nonnegative convex and quasiconvex functions. Since then numerous articles have appeared in the literature reflecting further applications in this category; see $[1,8,11,13]$ and references therein.

The main purpose of this paper is to establish new estimations and refinements of the Hermite-Hadamard inequality (1.1) for functions whose derivatives in absolute value are $P$-convex. Applications to the trapezoidal formula and special means are introduced.

\section{Hermite-Hadamard type inequality}

In this section we generalise Theorems $1.4-1.6$ with a $P$-convex function setting.

The next theorem gives a new result for the upper Hermite-Hadamard inequality for $P$-convex functions.

THEOREM 2.1. Let $f: I \rightarrow \mathbb{R}$ be a differentiable function on $I^{\circ}$ such that the function $\left|f^{\prime}\right|$ is $P$-convex. Suppose that $a, b \in I$ with $a<b$ and $f^{\prime} \in L_{1}[a, b]$. Then the following inequality holds:

$$
\left|\frac{f(a)+f(b)}{2}-\frac{1}{b-a} \int_{a}^{b} f(x) d x\right| \leq \frac{(b-a)\left(\left|f^{\prime}(a)\right|+\left|f^{\prime}(b)\right|\right)}{4} .
$$

Proof. By Lemma 1.1,

$$
\frac{f(a)+f(b)}{2}-\frac{1}{b-a} \int_{a}^{b} f(x) d x=\frac{b-a}{2} \int_{0}^{1}(1-2 t) f^{\prime}(t a+(1-t) b) d t .
$$

Since $\left|f^{\prime}\right|$ is $P$-convex, by (2.1),

$$
\begin{aligned}
\left|\frac{f(a)+f(b)}{2}-\frac{1}{b-a} \int_{a}^{b} f(x) d x\right| & =\left|\frac{b-a}{2} \int_{0}^{1}(1-2 t) f^{\prime}(t a+(1-t) b) d t\right| \\
& \leq \frac{(b-a)}{2} \int_{0}^{1}|1-2 t|\left|f^{\prime}(t a+(1-t) b)\right| d t \\
& =\frac{(b-a)\left(\left|f^{\prime}(a)\right|+\left|f^{\prime}(b)\right|\right)}{4} .
\end{aligned}
$$

This completes the proof.

The corresponding version for powers of the absolute value of the derivative is incorporated in the following result. 
Theorem 2.2. Let $f: I \rightarrow \mathbb{R}$ be a differentiable function on $I^{\circ}$. Assume that $p \in \mathbb{R}$, $p>1$, is such that the function $\left|f^{\prime}\right|^{p /(p-1)}$ is P-convex. Suppose that $a, b \in I$ with $a<b$ and $f^{\prime} \in L_{1}[a, b]$. Then the following inequality holds:

$$
\begin{aligned}
& \left|\frac{f(a)+f(b)}{2}-\frac{1}{b-a} \int_{a}^{b} f(x) d x\right| \\
& \quad \leq \frac{(b-a)\left(\left|f^{\prime}(a)\right|^{p /(p-1)}+\left|f^{\prime}(b)\right|^{p /(p-1)}\right)^{(p-1) / p}}{2(p+1)^{1 / p}} .
\end{aligned}
$$

Proof. Suppose that $a, b \in I$. By assumption, Hölder's inequality and Lemma 1.1,

$$
\begin{aligned}
\mid \frac{f(a)}{2}+f(b) & -\frac{1}{b-a} \int_{a}^{b} f(x) d x \mid \\
& \leq \frac{b-a}{2} \int_{0}^{1}|1-2 t|\left|f^{\prime}(t a+(1-t) b)\right| d t \\
& \leq \frac{b-a}{2}\left(\int_{0}^{1}|1-2 t|^{p} d t\right)^{1 / p}\left(\int_{0}^{1}\left|f^{\prime}(t a+(1-t) b)\right|^{q} d t\right)^{1 / q} \\
& =\frac{b-a}{2(p+1)^{1 / p}}\left(\int_{0}^{1}\left|f^{\prime}(t a+(1-t) b)\right|^{q} d t\right)^{1 / q} \\
& \leq \frac{b-a}{2(p+1)^{1 / p}}\left(\int_{0}^{1}\left(\left|f^{\prime}(a)\right|^{q}+\left|f^{\prime}(a)\right|^{q}\right) d t\right)^{1 / q} \\
& =\frac{b-a}{2(p+1)^{1 / p}}\left(\left|f^{\prime}(a)\right|^{q}+\left|f^{\prime}(a)\right|^{q}\right)^{1 / q},
\end{aligned}
$$

where $q:=p /(p-1)$ and since $\int_{0}^{1}|1-2 t|^{p} d t=1 /(p+1)$.

A more general inequality using Lemma 1.1 is as follows.

Theorem 2.3. Let $f: I \rightarrow \mathbb{R}$ be a differentiable function on $I^{\circ}$. Assume that $q \in \mathbb{R}$, $q>1$, is such that $\left|f^{\prime}\right|^{q}$ is a P-convex function. Suppose that $a, b \in I$ with $a<b$ and $f^{\prime} \in L_{1}[a, b]$. Then the following inequality holds:

$$
\left|\frac{f(a)+f(b)}{2}-\frac{1}{b-a} \int_{a}^{b} f(x) d x\right| \leq \frac{(b-a)}{4}\left(\left|f^{\prime}(a)\right|^{q}+\left|f^{\prime}(a)\right|^{q}\right)^{1 / q} .
$$

Proof. Suppose that $a, b \in I^{\circ}$. By the $P$-convexity of $f$ and Lemma 1.1, and using the well-known power mean inequality,

$$
\begin{aligned}
& \left|\frac{f(a)+f(b)}{2}-\frac{1}{b-a} \int_{a}^{b} f(x) d x\right| \\
& \quad \leq \frac{b-a}{2} \int_{0}^{1}\left|1-2 t \| f^{\prime}(t a+(1-t) b)\right| d t
\end{aligned}
$$




$$
\begin{aligned}
& \leq \frac{b-a}{2}\left(\int_{0}^{1}|1-2 t|^{p} d t\right)^{1-1 / q}\left(\int_{0}^{1}|1-2 t|\left|f^{\prime}(t a+(1-t) b)\right|^{q} d t\right)^{1 / q} \\
& =\frac{b-a}{4}\left(\int_{0}^{1}\left|f^{\prime}(t a+(1-t) b)\right|^{q} d t\right)^{1 / q} \\
& \leq \frac{b-a}{4}\left(\left|f^{\prime}(a)\right|^{q}+\left|f^{\prime}(a)\right|^{q}\right)^{1 / q} .
\end{aligned}
$$

This completes the proof.

\section{An extension to functions of several variables}

In this section some Hermite-Hadamard inequalities for functions of several variables on convex subsets of $\mathbb{R}^{n}$ will be given. First we introduce the notion of $P$-convexity for functions on a convex subset of $\mathbb{R}^{n}$.

Definition 3.1. The function $f: U \rightarrow \mathbb{R}$ is said to be $P$-convex on $U$ if it is nonnegative and, for all $x, y \in U$ and $\lambda \in[0,1]$, satisfies the inequality

$$
f(\lambda x+(1-\lambda) y) \leq f(x)+f(y) .
$$

The following proposition will be used throughout this section.

Proposition 3.2. Let $U \subseteq \mathbb{R}^{n}$ be a convex subset of $\mathbb{R}^{n}$ and $f: U \rightarrow \mathbb{R}$ be a function. Then $f$ is $P$-convex on $U$ if and only if, for every $x, y \in U$, the function $\varphi:[0,1] \rightarrow \mathbb{R}$, defined by

$$
\varphi(t):=f((1-t) x+t y)
$$

is $P$-convex on $I$ with $I=[0,1]$.

Proof. Let $x, y \in U$ be fixed. Assume that the function $\varphi$ is $P$-convex on $I$ with $I=[0,1]$. Suppose that $\lambda \in[0,1]$. Then

$$
\begin{aligned}
f((1-\lambda) x+\lambda y) & =\varphi(\lambda)=\varphi((1-\lambda) \cdot 0+\lambda \cdot 1) \\
& \leq \varphi(0)+\varphi(1)=f(x)+f(y) .
\end{aligned}
$$

It follows that $f$ is $P$-convex on $U$. Conversely, let $f$ be $P$-convex on $U$. Fix $x, y \in U$ and $t_{1}, t_{2} \in[0,1]$. Set $z_{1}:=\left(1-t_{1}\right) x+t_{1} y$ and $z_{2}:=\left(1-t_{2}\right) x+t_{2} y$. Then, for every $\lambda \in[0,1]$,

$$
\begin{aligned}
\varphi\left((1-\lambda) t_{1}+\lambda t_{2}\right) & =f\left(\left[1-(1-\lambda) t_{1}-\lambda t_{2}\right] x+\left[(1-\lambda) t_{1}-\lambda t_{2}\right] y\right) \\
& =f\left((1-\lambda) z_{1}+\lambda z_{2}\right) \\
& \leq f\left(z_{1}\right)+f\left(z_{2}\right) \\
& =\varphi\left(t_{1}\right)+\varphi\left(t_{2}\right) .
\end{aligned}
$$

Therefore, $\varphi$ is $P$-convex on $I$ with $I=[0,1]$. 
The following theorem is a generalisation of [10, Proposition 1].

Theorem 3.3. Let $U \subseteq \mathbb{R}^{n}$ be an open convex subset of $\mathbb{R}^{n}$. Assume that $f: U \rightarrow \mathbb{R}$ is a differentiable $P$-convex function on $U$. Then, for every $x, y \in S$ and every $a, b \in[0,1]$ with $a<b$, the following inequality holds:

$$
\begin{aligned}
\mid \frac{1}{2} \int_{0}^{a} f((1-s) x+s y) d s+\frac{1}{2} \int_{0}^{b} f((1-s) x+s y) d s \\
\quad-\frac{1}{b-a} \int_{a}^{b}\left(\int_{0}^{s} f((1-\theta) x+\theta y) d \theta\right) d s \mid \\
\quad \leq \frac{b-a}{4} \max \{f((1-a) x+a y), f((1-b) x+b y)\} .
\end{aligned}
$$

Proof. Let $x, y \in S$ and $a, b \in(0,1)$ with $a<b$. Since $f$ is a $P$-convex function, by Proposition 3.2 the function $\varphi:[0,1] \rightarrow \mathbb{R}^{+}$defined by

$$
\varphi(t):=f((1-t) x+t y)
$$

is $P$-convex on $I$ with $I=[0,1]$. Define the function $\phi:[0,1] \rightarrow \mathbb{R}^{+}$by

$$
\phi(t):=\int_{0}^{t} \varphi(s) d s=\int_{0}^{t} f((1-s) x+s y) d s .
$$

Obviously, for every $t \in(0,1)$,

$$
\phi^{\prime}(t)=\varphi(t)=f((1-t) x+t y) \geq 0 .
$$

Hence $\left|\phi^{\prime}(t)\right|=\phi^{\prime}(t)$. Applying Theorem 2.1 to the function $\phi$ implies that

$$
\left|\frac{\phi(a)+\phi(b)}{2}-\frac{1}{b-a} \int_{a}^{b} \phi(s) d s\right| \leq \frac{(b-a)\left[\phi^{\prime}(a)+\phi^{\prime}(b)\right]}{4},
$$

and we deduce that (3.1) holds.

\section{Applications to the trapezoidal formula}

Assume that $\Delta$ is a division of the interval $[a, b]$ such that

$$
\Delta: a=x_{0}<x_{1}<\cdots<x_{n-1}<x_{n}=b .
$$

For a given function $f:[a, b] \rightarrow \mathbb{R}$ we consider the trapezoidal formula

$$
T(f, \Delta)=\sum_{i=0}^{n-1} \frac{f\left(x_{i}\right)-f\left(x_{i+1}\right)}{2}\left(x_{i+1}-x_{i}\right) .
$$

It is well known that if $f$ is twice differentiable on $(a, b)$ and

$$
M=\sup _{x \in(a, b)}\left|f^{\prime \prime}(x)\right|<\infty,
$$


then

$$
\int_{a}^{b} f(x) d x=T(f, \Delta)+E(f, d)
$$

where the approximation error $E(f, d)$ of the integral $\int_{a}^{b} f(x) d x$ by $T(f, \Delta)$ satisfies

$$
|E(f, d)| \leq \frac{M}{12} \sum_{i=0}^{n-1}\left(x_{i+1}-x_{i}\right)^{3}
$$

Clearly, if the function $f$ is not twice differentiable or the second derivative is not bounded on $(a, b)$, then (4.1) does not hold true. In that context, the following result is important in order to obtain some estimates of $E(f, d)$.

Theorem 4.1. Let $f: I \rightarrow \mathbb{R}$ be a differentiable function such that $\left|f^{\prime}\right|$ is P-convex. Suppose that $a, b \in I$ with $a<b$ and $f^{\prime} \in L_{1}[a, b]$. Then, for every division $\Delta$ of the interval $[a, b]$,

$$
\left|T(f, \Delta)-\int_{a}^{b} f(x) d x\right|=\frac{\left|f^{\prime}(a)\right|+\left|f^{\prime}(b)\right|}{2} \sum_{i=0}^{n-1}\left(x_{i+1}-x_{i}\right)^{2} .
$$

Proof. Applying Theorem 2.1 on the subinterval $\left[x_{i}, x_{i}\right](i=0, \ldots, n-1)$ of the division $\Delta$ and adding from $i=0$ to $i=n-1$, we deduce that

$$
\left|T(f, \Delta)-\int_{a}^{b} f(x) d x\right|=\frac{1}{4} \sum_{i=0}^{n-1}\left(x_{i+1}-x_{i}\right)^{2}\left(f^{\prime}\left(x_{i}\right)+f\left(x_{i+1}^{\prime}\right)\right) .
$$

On the other hand, for every $x_{i} \in[a, b]$ there exists $\alpha_{i} \in[0,1]$ such that

$$
x_{i}=\alpha_{i} a+\left(1-\alpha_{i}\right) b
$$

By the $P$-convexity of $\left|f^{\prime}\right|$,

$$
\left|f^{\prime}\left(x_{i}\right)\right| \leq\left|f^{\prime}(a)\right|+\left|f^{\prime}(b)\right|
$$

Thus

$$
\left|f^{\prime}\left(x_{i}\right)+f\left(x_{i+1}^{\prime}\right)\right| \leq 2\left(\left|f^{\prime}(a)\right|+\left|f^{\prime}(b)\right|\right) .
$$

Therefore, combining relations (4.3) and (4.4) implies that (4.2) holds true and the proof is complete.

\section{Applications to special means}

We now give applications of our theorems to some special means of real numbers. We consider the following means for arbitrary real numbers $\alpha, \beta(\alpha \neq \beta)$. 
(1) Arithmetic mean:

$$
A(\alpha, \beta)=\frac{\alpha+\beta}{2}, \quad \alpha, \beta \in \mathbb{R}
$$

(2) Logarithmic mean:

$$
L(\alpha, \beta)=\frac{\alpha-\beta}{\ln |\alpha|-\ln |\beta|}, \quad|\alpha| \neq|\beta|, \alpha, \beta \neq 0, \alpha, \beta \in \mathbb{R} .
$$

(3) Generalised logarithmic mean:

$$
L_{n}(\alpha, \beta)=\left(\frac{\beta^{n+1}-\alpha^{n+1}}{(n+1)(\beta-\alpha)}\right)^{1 / n}, \quad n \in \mathbb{N}, \alpha, \beta \in \mathbb{R}, \alpha \neq \beta .
$$

Using the results of Section 2, we have the following propositions.

Proposition 5.1. Let $a, b \in \mathbb{R}, a<b$, and $n \in \mathbb{N}, n \geq 2$. Then

$$
\left|L_{n}^{n}(a, b)-A\left(a^{n}, b^{n}\right)\right| \leq \frac{n(b-a)}{4}\left(|a|^{n-1}+|b|^{n-1}\right) .
$$

Proof. The assertion follows from Theorem 2.1 applied to the function $f(x)=x^{n}$, $x \in \mathbb{R}$, because $\left|f^{\prime}\right|$ is $P$-convex.

Proposition 5.2. Let $a, b \in \mathbb{R}$, where $a<b$ and $0 \notin[a, b]$. Then

$$
\left|L^{-1}(a, b)-A\left(a^{-1}, b^{-1}\right)\right| \leq \frac{b-a}{4}\left(|a|^{-2}+|b|^{-2}\right) .
$$

Proof. The assertion follows from Theorem 2.1 applied to the function $f(x)=1 / x$, $x \in[a, b]$, because $\left|f^{\prime}\right|$ is $P$-convex.

Proposition 5.3. Let $a, b \in \mathbb{R}, a<b$, and $n \in \mathbb{N}, n \geq 2$. Then, for all $p>1$,

$$
\left|L_{n}^{n}(a, b)-A\left(a^{n}, b^{n}\right)\right| \leq \frac{(b-a)\left(|a|^{-2 p / p-1}+|b|^{-2 p / p-1}\right)^{(p-1) / p}}{2(p+1)^{1 / p}} .
$$

Proof. The assertion follows from Theorem 2.2 applied to the function $f(x)=1 / x$, $x \in \mathbb{R}$, because for all $p>1$ the function $\left|f^{\prime}\right|^{p /(p-1)}$ is $P$-convex.

\section{References}

[1] A. O. Akdemir and M. E. Özdemir, 'Some Hadamard-type inequalities for coordinated $P$-convex functions and Godunova-Levin functions', AIP Conf. Proc. 1309 (2010), 7-15.

[2] A. Barani, A. G. Ghazanfari and S. S. Dragomir, 'Hermite-Hadamard inequality through prequasiinvex functions', RGMIA Res. Rep. Coll. 14 (2011), article 48. 
[3] A. Barani, S. Barani and S. S. Dragomir, 'Refinements of Hermite-Hadamard type inequality for functions whose second derivatives absolute values are quasiconvex', RGMIA Res. Rep. Coll. 14 (2011), article 69.

[4] S. S. Dragomir, 'Two mappings on connection to Hadamard's inequality', J. Math. Anal. Appl. 167 (1992), 49-56.

[5] S. S. Dragomir, 'On Hadamard's inequalities for convex functions', Mat. Balkanica 6 (1992), 215-222.

[6] S. S. Dragomir, J. E. Pečarić and J. Sandor, 'A note on the Jensen-Hadamard inequality', Anal. Num. Theor. Approx. 19 (1990), 29-34.

[7] S. S. Dragomir, J. E. Pečarić and L. E. Persson, 'Some inequalities of Hadamard type', Soochow J. Math. 21 (1995), 335-341.

[8] S. S. Dragomir and C. E. M. Pearce, 'Quasiconvex functions and Hadamard's inequality', Bull. Aust. Math. Soc. 57 (1998), 377-385.

[9] S. S. Dragomir and R. P. Agarwal, 'Two inequalities for differentiable mappings and applications to special means of real numbers and to trapezoidal formula', Appl. Math. Lett. 11 (1998), 91-95.

[10] D. A. Ion, 'Some estimates on the Hermite-Hadamard inequality through quasiconvex functions', Ann. Univ. Craiova Math. Comp. Sci. Ser. 34 (2007), 82-87.

[11] M. E. Özdemir and C. Yıldız, 'New inequalities for Hermite-Hadamard and Simpson type and applications', arXiv:1103.1965v1 [math.CA] 10 Mar 2011.

[12] J. Pečarić, F. Proschan and Y. L. Tong, Convex Functions, Partial Ordering and Statistical Applications (Academic Press, New York, 1991).

[13] K. L. Tseng, G. S. Yang and S. S. Dragomir, 'On quasiconvex functions and Hadamard's inequality', RGMIA Res. Rep. Coll. 14 (2003), article 1.

\title{
A. BARANI, Department of Mathematics, Lorestan University, PO Box 465, Khoramabad, Iran \\ e-mail: alibarani2000@yahoo.com,barani.a@lu.ac.ir
}

\author{
S. BARANI, Department of Civil Engineering, Shahid Chamran University, \\ PO Box 135, Ahvaz, Iran \\ e-mail: seebb86@yahoo.com
}

